Pacific Journal of Mathematic 


\section{THE EXTENSION OF BILINEAR FUNCTIONALS}

\section{T. L. HAYDEN}

Using the relationship between bilinear functionals and linear operators we obtain some theorems on the extension of bilinear functionals. To extend bilinear functionals in Hilbert Spaces a special constructional process is given which is a generalization of the usual inner product. This allows the construction of bilinear functionals with special properties. In particular it allows a generalization of the Lax-Milgram Theorem. We also extend the Lax-Milgram Theorem to reflexive Banach Spaces.

In order to fix the terminology, let $U$ and $V$ be Banach Spaces (real or complex), then by a bilinear functional we mean a function $F$ from $U \times V$ to the complex (or real) numbers such that $F(u, v)$ is linear on $U$ for each fixed $v \in V$ and vice versa. The norm of a bounded bilinear functional $F$, denoted by $\|F\|$, is defined as:

$$
\|F\|=\inf \{K>0:|F(u, v)| \leqq K\|u\|\|v\| \text { for all } u \in U, v \in V\} .
$$

Hence a bounded bilinear functional is jointly continuous on $U \times V$ in the product topology and we note that:

$$
\|F\|=\sup _{\substack{\|x\| \leq 1 \\\|y\| \leq 1}}|F(x, y)|=\sup _{\substack{\|x\|=1 \\\|y\|=1}}|F(x, y)| \leqq \sup _{\|x\|\|y\|=1}|F(x, y)| \leqq\|F\| .
$$

If $S$ and $T$ are subspaces of $U$ and $V$ respectively and $B_{0}$ is a bounded bilinear functional on $S \times T$, then we call $B$ an extension of $B_{0}$ to $U \times V$ if $B$ is a bounded bilinear functional on $U \times V$ such that $B_{0}(s, t)=B(s, t)$ on $S \times T$ and $\left\|B_{0}\right\|=\|B\|$. If such an extension exists we shall say that $B_{0}$ can be extended to $U \times V$. Furthermore, if each bounded bilinear functional on $S \times T$ can be extended to $U \times V$ we will say that $S$ and $T$ have the bilinear extension property.

\section{Some extension theorems.}

Theorem 1. Suppose $U, V, W$ are normed linear spaces and $S$ and $T$ are subsets of $U$ and $V$ respectively. A necessary and sufficient condition to extend a bounded bilinear operator $B_{0}$ from $S \times T$ into $W$ to a bounded bilinear operator $B$ from (Span $S) \times$ (Span $T$ ) into $W$ is that there exists a constant $c$ such that

$$
\left\|\sum_{k, j} \alpha_{k} \beta_{j} B_{0}\left(x_{k}, y_{j}\right)\right\| \leqq c\left\|\sum_{k} \alpha_{k} x_{k}\right\|\left\|\sum_{j} \beta_{j} y_{j}\right\| \text {. }
$$


For each finite subset $x_{1}, \cdots, x_{n} \in S$ and $y_{1}, \cdots, y_{m} \in T$ and every choice of scalars $\alpha_{k}$ and $\beta_{\jmath}$.

The proof is similar to the proof of the linear case which is found for example in [3, p. 127]. That one can extend bounded bilinear functionals to the closure or completion of a space has a proof similar to the linear case. For one such version for bilinear functionals see $[1$, p. 105]. Unless it is specifically mentioned we will not assumed that the subspaces in question in each theorem are necessarily closed. Hence in each such theorem one should first extend to the closure and then proceed with the proof.

Our next two theorems show the relation of extending bilinear functionals and the extension of linear operators. One of the best references to the problem of extending linear operators is Nachbin [6]. (See also [5]). References and additional information to the following facts may be found in [6].

We shall say that a Banach Space $U$ has property $E$ (the extension property of type $\infty$ in the terminology of [6]) if for any Banach Space $V$, any closed subspace $S$ of $V$, and any continuous linear operator $F_{0}$ from $S$ into $U$, there exists a continuous linear extension $F$ of $F_{0}$ to $V$ with values in $U$ and the same norm. The classical Hahn-Banach theorem says $U$ has property $E$ if its dimension is one. A Banach Space $U$ has property $E$ if and only if for any Banach Space $W$ containing $U$ as a Banach subspace there is a projection of norm one of $W$ onto $U$.

Also the topological dual $U^{*}$ of a Banach Space $U$ has property $E$ if and only if $U$ is metrically isomorphic to a space $L^{1}(\mu)$ of all real integrable functions with respect to a suitable positive measure $\mu$ on a locally compact space [6, p. 345].

Theorem 2. If $U$ and $V$ are Banach Spaces and $S$ and $T$ are subspaces of $U$ and $V$ respectively such that $T^{*}$ (the topological dual of $T$ ) and $U^{*}$ have property $E$, then each bounded bilinear functional on $S \times T$ can be extended to $U \times V$.

Proof. Let $B_{0}$ be a bounded bilinear functional on $S \times T$ with norm $\left\|B_{0}\right\|$. If we fix $s \in S$, then $B(s, t)=F_{s}(t)$ is a bounded linear functional on $T$, i.e. $F_{s}(t) \in T^{*}$. Let us denote the value of a linear functional $F$ at $x$ by $\langle x, F\rangle$. If $G_{0}$ is the map from $S$ into $T^{*}$ such that $G_{0}(s)=F_{s}$, then $G_{0}$ is a bounded linear operator from $S$ into $T^{*}$ and $\left\|G_{0}\right\|=\left\|B_{0}\right\|$. The linearity of $G_{0}$ follows from the relation $\left\langle t, G_{0}(s)\right\rangle=B_{0}(s, t)$ and the bilinearity of $B$, and

$$
\left\|G_{0}\right\|=\sup _{\|s\| \leqq 1}\left\|G_{0}(s)\right\|=\sup _{\substack{\|t\| \leq 1 \\\|s\| \leq 1}}\left|\left\langle t, G_{0}(s)\right\rangle\right|=\sup _{\substack{\|t\| \leq 1 \\\|s\| \leq 1}}\left|B_{0}(s, t)\right|=\left\|B_{0}\right\| .
$$


Since $T^{*}$ has property $E$ we extend $G_{0}$ to a linear operator $G$ on $U$ into $T^{*}$ with the same norm. Now let $B(u, t)=\langle t, G(u)\rangle$ for $(u, t) \in U \times T$ and it is easy to see that $B$ is an extension of $B_{0}$ to $U \times T$. Using a similar procedure for the spaces $U^{*}$ and $T$ we obtain the desired extension to $U \times V$.

One natural way to try to extend bilinear functionals is to factor through the bilinear functional on $S \times T$ into a linear functional on the tensor product $S \otimes T$. Now the bounded bilinear functionals on $S \times T$ are isometric to the bounded linear functionals on $S \otimes T$ under the greatest cross norm topology on $S \otimes T$. (See Grothendieck, Produits Tensoriels Topologiques Et Espaces Nucleaires, Memoirs of A.M.S. No. 16, for related statements in topological vector spaces.) Hence if we extend the linear functional on $S \otimes T$ to $U \otimes V$ we have an extension of the bilinear functional on $S \times T$ to $U \times V$. However, Schatten [7, p. 57] has shown that the greatest cross norm topology on $U \otimes V$ is not an extension of the greatest cross norm topology on $S \otimes T$ in general. The next few theorems are closely related to those obtained by Schatten in proving this result.

Theorem 3. Suppose $U$ and $V$ are Banach Spaces with the bilinear extension property on the subspaces $S$ and $T$, then every bounded linear operator from $S$ into $T^{*}\left(T\right.$ into $\left.S^{*}\right)$ can be extended to a linear operator from $U$ into $T^{*}\left(V\right.$ into $\left.T^{*}\right)$ with the same norm.

Proof. Suppose $F_{0}$ is a bounded linear operator from $S$ into $T^{*}$. Then there exists, as in the proof of Theorem 2, a bounded bilinear functional $B_{0}$ on $S \times T$ such that $B_{0}(s, t)=\left\langle t, F_{0}(s)\right\rangle$ and $\left\|B_{0}\right\|=\left\|F_{0}\right\|$. Since $S$ and $T$ have the extension property we may extend $B_{0}$ to a bounded bilinear functional $B$ on $U \times V$, such that $\|B\|=\left\|B_{0}\right\|$. We may restrict $B$ to $U \times T$ and find an $F$ such that $B(u, t)=\langle t, F(u)\rangle$ on $U \times T$ with $\|F\|=\left\|F_{0}\right\|$ and $F$ restricted to $S$ is $F_{0}$. Hence $F$ is the desired extension of $F_{0}$.

We also note that we could consider the set $U \times V$ and by a similar process we have an extension of $F_{0}$ to $U$ but with final values in $V^{*}$.

THEOREM 4. Suppose in addition to the hypothesis of Theorem 3 that $T^{*}=S$, then there is a projection of norm one of $U$ onto $S$.

Proof. Let $F_{0}$ be the identity operator from $S$ into $T^{*}$. As in the proof of Theorem 3 associate $B_{0}$ with $F_{0}$ and $\left\|B_{0}\right\|=1$. The extension then of $F_{0}$ to $F$ gives us a linear operator of norm one of $U$ 
into $T^{*}$ or $S$ which is the identity on $S$, i.e., $F$ is a projection of norm one.

Theorem 5. Let $S$ be a subspace of the Banach Space $U$, then the existence of a projection of $U$ on $S$ of norm one implies that if $V$ is a Banach Space then every bounded bilinear form on $S \times V$ can be extended to $U \times V$.

Proof. Let $B_{0}$ be a bounded linear operator on $S \times V$, and let $P$ be a projection of $U$ onto $S$ with norm one. It is clear that $B(u, v)=B_{0}(P u, v)$ is the desired extension.

Theorem 6. Suppose $S$ is a subspace of the Banach Space U, and that $S$ is the topological dual of a Banach Space T, i.e. $S=T^{*}$, and that every bounded bilinear functional on $S \times T$ can be extended to $U \times T$, then if $V$ is a Banach Space every bilinear functional on $S \times V$ can be extended to $U \times V$.

Proof. By Theorem 4 there is a projection of norm one of $U$ onto $S$ and hence by Theorem 5 the result follows.

CoRollary 1. If $S$ is a subspace of Hilbert Space $U$ then for every Banach Space $V$ and every bounded bilinear functional $B$ on $S \times V$ there is an extension to $U \times V$.

Corollary 2. If $U$ and $V$ are Banach Spaces and $S$ and $T$ are subspaces of $U$ and $V$ respectively and there exists a projection of norm one of $U$ on $S$ and a projection of norm one of $V$ on $T$ then every bounded bilinear functional on $S \times T$ can be extended to $U \times V$.

Corollary 3. A Banach Space $U$ is a Hilbert Space if and only if every bounded bilinear functional on $S \times S^{*}$ can be extended to $U \times S^{*}$ for any two dimensional subspace $S$ of $U$.

Proof. By Theorem 4 we have a projection of norm one on each two dimensional subspace, which implies that $U$ is a Hilbert Space.

Although Corollary 2 shows that bilinear functionals on subspaces of Hilbert Spaces may always be extended, we repeat another proof given in [2] whose explicit construction yields other results immediately.

THEOREM 7. Suppose $S_{1}, S_{2}, \cdots, S_{n}$ are subspaces respectively of the complex Hilbert Spaces $X_{1}, X_{2}, \cdots, X_{n}$ and $f\left(s_{1}, s_{2}, \cdots, s_{n}\right)$ is a 
multilinear functional defined on $\mathbf{X}_{k=1}^{n} S_{k}$ with norm $K$. Then there exists a multilinear functional $F$ on $\mathbf{X}_{k=1}^{n} X_{k}$ with norm $K$ such that $F$ is $f$ on $\mathbf{X}_{k=1}^{n} S_{k}$.

Proof. First we show that $f$ can be extended to a function defined on $\mathrm{X}_{k=1}^{n}\left(S_{k} \cup\left\{x_{k}\right\}\right)$ for $x_{k} \in X_{k}, k=1,2, \cdots, n$. In fact, let $x_{k} \perp S_{k}$, and define

$$
h\left(a_{1} x_{1}+s_{1}, \cdots, a_{n} x_{n}+s_{n}\right)=K \prod_{k=1} a_{k}\left\|x_{k}\right\|+f\left(s_{1}, \cdots, s_{n}\right)
$$

where $a_{1}, a_{2}, \cdots, a_{n}$ are scalars and $K$ is the norm of $f$. It is immediate that the extension is multilinear, and we now show that the norm is the same.

First we show by induction that

$$
\left(\prod_{k=1}^{n}\left\|a_{k} x_{k}\right\|+\prod_{k=1}^{n}\left\|s_{k}\right\|\right) \leqq \prod_{k=1}^{n}\left(\left\|a_{k} x_{k}\right\|^{2}+\left\|s_{k}\right\|^{2}\right)^{1 / 2} \quad \text { for } \quad n \geqq 2 .
$$

For $n=2$ this is Cauchy's inequality. Suppose it is true for $n=l-1$, then

$$
\begin{aligned}
\left(\prod_{k=1}^{l}\left\|a_{k} x_{k}\right\|+\prod_{k=1}^{l}\left\|s_{k}\right\|\right) & \leqq\left(\prod_{k=1}^{l-1}\left\|a_{k} x_{k}\right\|+\prod_{k=1}^{l-1}\left\|s_{k}\right\|\right)\left(\left\|a_{l} x_{l}\right\|^{2}+\left\|s_{l}\right\|^{2}\right)^{1 / 2} \\
& \leqq \prod_{k=1}^{l-1}\left(\left\|a_{k} x_{k}\right\|^{2}+\left\|s_{k}\right\|^{2}\right)^{1 / 2}\left(\left\|a_{l} x_{l}\right\|^{2}+\left\|s_{l}\right\|^{2}\right)^{1 / 2} \\
& \leqq \prod_{k=1}^{l}\left(\left\|a_{k} x_{k}\right\|^{2}+\left\|s_{k}\right\|^{2}\right)^{1 / 2} .
\end{aligned}
$$

Hence

$$
\begin{gathered}
\left|h\left(a_{1} x_{1}+s_{1}, \cdots, a_{n} x_{n}+s_{n}\right)\right| \leqq K\left(\prod_{k=1}^{n}\left\|a_{k} x_{k}\right\|+\prod_{k=1}^{n}\left\|s_{k}\right\|\right) \\
\leqq K \prod_{k=1}^{n}\left(\left\|a_{k} x_{k}\right\|^{2}+\left\|s_{k}\right\|^{2}\right)^{1 / 2}=K \prod_{k=1}^{n}\left\|a_{k} x_{k}+s_{k}\right\| .
\end{gathered}
$$

The remainder of the proof follows from a straightforward application of Zorn's Lemma similar to that in the Hahn-Banach Theorem.

3. Corollaries. We will suppose in this section that $U$ and $V$ are Hilbert Spaces and $S$ and $T$ are subspaces of $U$ and $V$ respectively. From the proof of Theorem 7 we obtain the following

Conollary 4. Suppose $S \subset U$ and $B$ is a bilinear functional on $S \times S$ such that:

(i) $|B(s, t)| \leqq K_{1}\|s\|\|t\|$ for each $s$, $t$ in $S$ and

(ii) $|B(s, s)| \geqq K_{2}\|s\|^{2}$ for each $s$ in $S$,

Then $B$ may be extended to $U \times U$ such that (i) and (ii) hold on $U \times U$. 
One also can easily see that a bounded symmetric bilinear functional on $S \times S(B(u, v)=B(v, u))$ can be extended to a symmetric bilinear functional on $U \times U$. Furthermore a bounded sesquilinear functional on $S \times T$ can be extended to $U \times V$. One simply uses the fact that the sesquilinear form on $S \times T$ is a bilinear form on $S \times T^{\prime}$ where $T^{\prime}$ is the so called complex conjugate of $T$.

The proofs of the following corollaries follow from the extension theorem and since they are similar to the proofs in the linear case, they will be omitted.

COROLlaRY 5. If $u \neq 0$ and $v \neq 0$ are in $U$ and $V$ respectively then there exists a bilinear functional $B$ on $U \times V$ such that $B(u, v)=\|u\|\|v\|$ and $\|B\|=1$.

Corollary 6. Suppose $x_{0} \in U$ and distance from $x_{0}$ to $S$ is $d_{1}>0$ and $y_{0} \in V$ such that the distance from $y_{0}$ to $T$ is $d_{2}>0$. Then there exists a bilinear functional $B$ of norm one on $U \times V$ such that $B(s, t)=0$ for $(s, t) \in S \times T$ and $B\left(x_{0}, y_{0}\right)=d_{1} d_{2}$.

Corollary 7. Let $E \subset U, F \subset V . \quad A$ necessary and sufficient condition that $\left(x_{0}, y_{0}\right) \in U \times V$ to belong to (closure span $\left.E\right) \times($ closure span $F)$ is that $B\left(x_{0}, y_{0}\right)=0$ for every bounded linear functional which vanishes on $E \times F$.

The next corollary follows from Theorem 1 and the extension theorem and indicates when one can solve infinite systems of equations in bilinear functionals.

Corollary 8. Let $M$ and $N$ be indexing sets. Let $\left\{\left(u_{\alpha}, v_{\beta}\right)\right.$; $(\alpha, \beta) \in M \times N\} \subset U \times V$ and $\left\{C_{\alpha \beta} ;(\alpha, \beta) \in M \times N\right\} \subset$ complex numbers. $A$ necessary and sufficient condition for the existence of a bounded bilinear functional $B$ on $U \times V$ such that

(i) $B\left(u_{\alpha}, v_{\beta}\right)=C_{\alpha \beta}$ for each $(\alpha, \beta) \in M \times N$ and

(ii) $\|B\| \leqq K$

is that

$$
\left|\sum d_{\alpha} e_{\beta} C_{\alpha \beta}\right| \leqq K\left\|\sum d_{\alpha} u_{\alpha}\right\|\left\|\sum e_{\beta} v_{\beta}\right\|
$$

hold for every finite subset of $M \times N$ and every choice of scalars $d_{\alpha}$ and $e_{\beta}$.

We note that if $F$ and $G$ are bounded linear functionals on $S$ and $T$ respectively then the bilinear functional defined by $B(s, t)=F(s) G(t)$ is bounded on $S \times T$ and can obviously be extended. On the other hand a nondegenerate bilinear functional on $U \times V$ can not be re- 
presented in a form with the variables separated. In fact we have the following

Theorem 8. Suppose $X$ and $Y$ are normed linear spaces and $B$ is a bounded bilinear functional on $X \times Y$ such that $B(x, y)=0$ for all $y \in Y$ implies that $x$ is zero. Then $B$ can not be factored as $B(x, y)=\sum_{i=1}^{n} F_{i}(x) G_{i}(y)$ where $F_{i} \in X^{*}, G_{i} \in Y^{*}$ for $1 \leqq i \leqq n$, and $n<\operatorname{dim} X$.

Proof. Suppose the conclusion is false. Let $K_{1}=$ Kernal $F_{i}$, $1 \leqq i \leqq n$. Since $K_{\imath}$ is a maximal closed subspace of $X$, hence $\operatorname{dim} \bigcap_{i=1}^{n} K_{i} \neq 0$. But for $x \in \bigcap_{i=1}^{n} K_{i}, B(x, y)=0$ for all $y \in Y$.

It is known that if there exists a nondegenerate bilinear functional on $U \times V$ where $U$ and $V$ are finite dimensional then $\operatorname{dim} U=$ $\operatorname{dim} V$.

THEorem 9. Suppose $X$ and $Y$ are normed linear spaces and $B$ is a nondegenerate bounded bilinear functional on $X \times Y$. Then $\operatorname{dim} X \leqq \operatorname{dim} Y^{*}$ and $\operatorname{dim} Y \leqq \operatorname{dim} X^{*}$.

Proof. Again as in the proof of Theorem 3 we associate a linear operator $A$ from $X$ into $Y^{*}$ with the bilinear functional $B$. Since $B$ is nondegenerate this implies that $A$ is one to one and the result follows.

CoRollary 9. If $U$ and $V$ are Hilbert Spaces and there exists a nondegenerate bounded bilinear functional on $U \times V$ then $\operatorname{dim} U=$ $\operatorname{dim} V$ where $\operatorname{dim} U$ and $\operatorname{dim} V$ may be either Hilbert Space dimension or Vector Space dimension.

4. An application. A variant of the F. Riesz representation, the Lax-Milgram Theorem [4], is useful in proving the existence of solutions of partial differential equations. Let $(\cdot, \cdot)$ denote the inner product in Hilbert Space.

THEOREM 10. (Lax-Milgram) Let $U$ be a Hilbert Space and $B$ a bounded sesqui-linear functional on $U \times U$ such that there exists a $\delta>0$ such that $B(u, u) \geqq \delta\|u\|^{2}$. Then there exists a unique bounded linear operator $T$ with a bounded inverse $T^{-1}$ such that $(u, v)=$ $B(u, T v)$ when $u, v \in U$ and $\|T\| \leqq 1 / \delta,\left\|T^{-1}\right\| \leqq\|B\|$.

Lemma. If $S$ is a subspace of a Hilbert Space $U$ and $B$ is a bounded sesquilinear functional on $S \times S$ such that $B(s, s) \geqq \delta\|s\|^{2}$ 
for some $\delta>0$, then $B$ can be extended to $U \times U$ with the same properties.

Proof. As in the proof of Theorem 5, let $x \perp S$ and define $\bar{B}$ on $(x \cup S) \times(x \cup S)$ by

$$
\bar{B}(\alpha x+s, \beta x+t)=\alpha \bar{\beta}\|B\|\|x\|^{2}+B(s, t) .
$$

As before we now observe that $\bar{B}$ is a sesqui-linear functional on $(x \cup S) \times(x \cup S)$ with norm $\|B\|$.

Now

$$
\begin{aligned}
\bar{B}(\alpha x+s, \alpha x+s) & =\|B\||\alpha|^{2}\|x\|^{2}+B(s, s) \\
& \geqq \delta\left(\|\alpha x\|^{2}+\|s\|^{2}\right) \\
& =\delta\|\alpha x+s\|^{2} .
\end{aligned}
$$

An application of Zorn's Lemma completes the proof. We note that no claim to the uniqueness of the extension is made.

This Lemma immediately gives us the following extension of the Lax-Milgram Theorem.

THEOREM 11. Let $S$ be a subspace of the Hilbert Space $U$ and $B$ a sesquilinear functional satisfying the hypothesis of the LaxMilgram Theorem. Then there is an extension of $B$ to $\bar{B}$ on $U \times U$ satisfying the hypothesis of Lax-Milgram Theorem and hence there $i$ an extension of the linear operator $T$ to $\bar{T}$ from $U$ onto $U$ which satisfies the conclusion of the Lax-Milgram Theorem.

We may also extend the Lax-Milgram Theorem in the following direction.

Theorem 12. Suppose $U$ and $V$ are Banach Spaces, $V$ is reflexive, and that $B$ is a bounded nondegenerate bilinear functional on $U \times V$. Then a necessary and sufficient condition that every bounded linear functional $F$ on $V$ have a unique representation of the form $F(v)=B(u, v)$ for some fixed $u \in U$ is that there exists an $m>0$ such that for each $u \in U$, $\sup _{\|v\|=1}|B(u, v)| \geqq m\|u\|$.

Proof. Suppose the representation holds, then $B$ induces a linear operator $A$ from $U$ into $V^{*}$ in the usual manner. Since $B$ is nondegenerate, $A$ is one-to-one and $\|A\|=\|B\|$. Since every linear functional in $V^{*}$ has this representation $A$ must be onto. Hence $A$ has a bounded inverse $A^{-1}$. However, a necessary and sufficient condition that $A$ have a bounded inverse is that there exists an $m>0$ such that $\|A(u)\| \geqq m\|u\|$. But 


$$
\|A(u)\|=\sup _{\|v\|=1}|\langle v, A(u)\rangle|=\sup _{\|v\|=1}|B(u, v)|,
$$

and it follows that the condition is necessary.

Now suppose that the condition is satisfied. Hence $A$ is one-toone and onto $R(A)$ which is closed in $V^{*}$. If $R(A) \neq V^{*}$ let $f^{*} \neq$ $0 \nexists R(A)$. By the Hahn Banach Theorem there exists $v^{* *} \in V^{* *}$ such that $v^{* *}\left(f^{*}\right)=1$ and $v^{* *}(R(A))=0$. Let $v \in V$ correspond to $v^{* *}(V$ is reflexive). Hence $f^{*}(v)=1$, and $B(u, v)=A(u)(v)=0$ for all $u$ which implies that $v=0$ since $B$ is nondegenerate. This contradiction shows $R(A)=V^{*}$.

REMARKs. Note that $\left\|A^{-1}\right\| \leqq 1 / m$. Also if there exists an $m_{1}>0$ such that for each $\|v\|=1$, $\sup _{\|u\|=1}|B(u, v)| \geqq m_{1}$, then this implies that the bilinear form is nondegenerate and hence the usual Lax-Milgram Lemma is a special case.

There are some corollaries to the Lax-Milgram Theorem due to Littman and Schechter [8, 568] which are useful in Elliptic Boundary Value Problems. The above theorem gives the following generalizations.

CoRollary. Suppose $B$ is a bounded, nondegenerate, bilinear functional on the Banach Spaces $U$ and $V$, and that $V$ is reflexive. If there exists an $m>0$ such that for each $u \in U, \sup _{\|v\| \mid=1}|B(u, v)|>$ $m\|u\|$, then for each proper closed subspace $K$ of $V$ there exists a $u \in U$ such that $B(u, v)=0$ for each $v$ in $K$.

Corollary. Suppose $U$ and $V$ are Banach Spaces, $V$ is reflexive and that $B$ is a bounded bilinear functional on $U \times V$. If there exist closed subspaces $S$ and $T$ of $U$ and $V$ respectively such that $B$ satisfies the hypothesis of the preceding corollary on $S \times T$, then for each $u \in U$, there is a unique $u^{*}$ in $S$ such that $B\left(u-u^{*}, T\right)=0$.

Proof. For a fixed $u, B(u, t)$ is a bounded linear functional $t^{*}$ on $T$. Therefore there is a unique $u^{*}$ in $S$ such that $B\left(u^{*}, t\right)=\left\langle t, t^{*}\right\rangle$ for all $t$ in $T$. Hence $B(u, t)=B\left(u^{*}, t\right)$ for all $t$ in $T$.

COROLLARY. Suppose the hypothesis of the preceding corollary holds, then for each $u \in U$ there is a unique $u^{*}$ in $S$ and $a u^{* *}$ such that $B\left(u^{* *}, t\right)=0$ for all $t \in T$ and $u=u^{*}+u^{* *}$.

The author appreciates the helpful suggestions of the referee and in particular wishes to give credit to the referee for the brief proof of Theorem 12. 


\section{REFERENCES}

1. R. C. Cooke, Linear Operators, MacMillan Co., London, 1953.

2. T. L. Hayden, A conjecture on the extension of bilinear functionals (to appear in the Amer. Math. Monthly)

3. L. V. Kantorovich and G. P. Akilov, Functional Analysis in Normed Spaces, MacMillan Co., New York 1964.

4. P. D. Lax and A. N. Milgram. Parabolic equations, Ann. of Math. 33 (1954), 167-190.

5. J. Lindenstrauss, On the extension of operators, Illinois J. Math. 8 (1964), 488-499.

6. L. Nachbin, Some problems in extending and lifting linear transformations, Proc. international symposium on linear spaces, Jerusalem, 1961, 340-352.

7. R. Schatten, A Theory of Cross Spaces, Annals of Math. Studies, No. 26, Princeton, 1950 .

8. M. Schechter, Remarks on elliptic boundary value problems, Comm. Pure Appl. Math. 12 (1959), 561-578.

Received August 3, 1966.

UNIVERSITY OF KENTUCKY

Lexington, KentuCky 


\section{PACIFIC JOURNAL OF MATHEMATICS}

\section{EDITORS}

\section{H. SAMELSON}

Stanford University

Stanford, California

J. P. JANS

University of Washington

Seattle, Washington 98105
J. Dugundji

University of Southern California Los Angeles, California 90007

RICHARD ARENS

University of California

Los Angeles, California 90024

\section{ASSOCIATE EDITORS}
E. F. BECKENBACH
B. H. NeumanN
F. WOLF
K. YosidA

\section{SUPPORTING INSTITUTIONS}

UNIVERSITY OF BRITISH COLUMBIA

CALIFORNIA INSTITUTE OF TECHNOLOGY

UNIVERSITY OF CALIFORNIA

MONTANA STATE UNIVERSITY

UNIVERSITY OF NEVADA

NEW MEXICO STATE UNIVERSITY

OREGON STATE UNIVERSITY

UNIVERSITY OF OREGON

OSAKA UNIVERSITY

UNIVERSITY OF SOUTHERN CALIFORNIA
STANFORD UNIVERSITY

UNIVERSITY OF TOKYO

UNIVERSITY OF UTAH

WASHINGTON STATE UNIVERSITY

UNIVERSITY OF WASHINGTON

AMERICAN MATHEMATICAL SOCIETY CHEVRON RESEARCH CORPORATION TRW SYSTEMS

NAVAL ORDNANCE TEST STATION 


\section{Pacific Journal of Mathematics \\ Vol. 22, No. $1 \quad$ January, 1967}

Charles A. Akemann, Some mapping properties of the group algebras of a

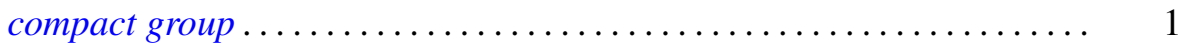

A. V. Boyd, Note on a paper by Uppuluri ..................... 9

Thomas Craig Brown, A semigroup union of disjoint locally finite subsemigroups which is not locally finite .....................

Richard Thomas Bumby and Everett C. Dade, Remark on a problem of

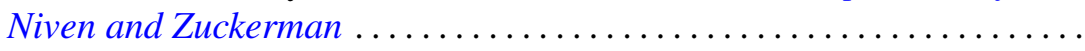

James Calvert, An integral inequality with applications to the Dirichlet problem .........................................

Jack Gary Ceder and Terrance Laverne Pearson, On products of maximally resolvable spaces ....................................

William Guignard Faris, The product formula for semigroups defined by

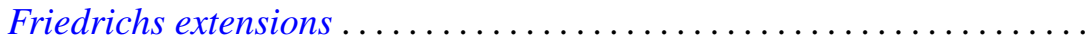

Robert S. Freeman, Closed operators and their adjoints associated with elliptic differential operators ........................ 71

Thomas Lee Hayden, The extension of bilinear functionals ............. 99

Gloria Conyers Hewitt, Limits in certain classes of abstract algebras . . . . . 109

Tilla Weinstein, The dilatation of some standard mappings ........... 117

Mitsuru Nakai, On Evans' kernel ......................... 125

Ernest Levane Roetman, On the biharmonic wave equation ............ 139

Malcolm Jay Sherman, Operators and inner functions ... . .

Walter Laws Smith, On the weak law of large numbers and the generalized elementary renewal theorem

A. J. Ward, On H-equivalence of uniformities: The Isbell-Smith problem 